\title{
CAROTID INTIMA MEDIA THICKNESS IN PATIENTS WITH ACUTE ISCHAEMIC STROKE
}

\author{
Suresh Muthezhathu Kesavadas ${ }^{1}$, Amy Susan George2 ${ }^{2}$ N. Roy ${ }^{3}$
}

1 Professor, Department of Internal Medicine, Government Medical College, Trivandrum.

2Junior Resident, Department of Internal Medicine, Government Medical College, Trivandrum.

3 Professor and HOD, Department of Radiodiagnosis, Government Medical College, Trivandrum.

\section{BACKGROUND}

ABSTRACT

Carotid intima media thickness reflects ongoing atherosclerosis in the body and can be measured by B-mode ultrasound. It is found to be a risk factor for cardiovascular diseases. Our study intends to find association of carotid intima media thickness with acute ischaemic stroke.

Objectives- To study the association between common carotid artery intima media thickness in patients with acute ischaemic stroke. To study the pattern between carotid intima media thickness and subtypes of ischaemic stroke. To study the pattern between carotid intima media thickness and size of infarct.

\section{MATERIALS AND METHODS}

The study design was a case control study. After getting ethical clearance, 42 cases and 42 controls satisfying the inclusion criteria were enrolled in the study. The data on demographic characteristics, smoking and cardiovascular risk factors were collected as a structured questionnaire. B Mode ultrasound Doppler of both common carotid arteries with carotid intima media thickness measurement was done for all participants in the study. Data analysis was done by SPSS software. Tests of significance were done to find association between CIMT and acute ischaemic stroke, subtypes of brain infarction, while scattered plot for assessing size of infarct.

\section{RESULTS}

Carotid plaques were present in $40.5 \%$ of cases and $14.3 \%$ of controls. The mean carotid intima media thickness in the present study is $0.90 \pm 0.1$ among cases and $0.80 \pm 0.1$ among controls ( $\mathrm{p}=0.001)$. The risk for stroke was $57.1 \%$ among cases and $23.8 \%$ among controls $(\mathrm{p}=0.002)$. Among infarct subtypes $52.3 \%$ had PACIs, $23.8 \%$ had LACIs, $16.6 \%$ had POCIs and $7 \%$ had TACIs. CIMT $>0.9 \mathrm{~mm}$ was found in $68.5 \%$ of PACI, $66.6 \%$ of TACI, $14.2 \%$ of POCI and $60 \%$ of LACI ( $\mathrm{p}=0.09$ ). There was no correlation between CIMT and size of infarct $(r=0.132, p=0.406)$.

\section{CONCLUSION}

There was significant association between CIMT and acute ischaemic stroke. No association was found between CIMT and brain infarction subtypes. No correlation was found between CIMT and size of infarct.

\section{KEYWORDS}

Carotid Intima Media Thickness, Stroke, Carotid Plaques.

HOW TO CITE THIS ARTICLE: Kesavadas SM, George AS, Roy N. Carotid intima media thickness in patients with acute ischaemic stroke. J. Evolution Med. Dent. Sci. 2018;7(07):914-918, DOI: 10.14260/jemds/2018/208

\section{BACKGROUND \\ Currently, ischaemic heart disease and stroke are the leading causes of mortality worldwide. The incidence of stroke increases with increasing age and with the growing elderly population worldwide the number of patients with stroke are likely to increase. Developing countries like India are facing a double burden of communicable and non-communicable diseases. Stroke is one of the leading causes of death and disability in India. Stroke is generally defined as sudden or rapidly developing clinical signs of focal disturbance of cerebral function lasting more than 24 hours or leading to death with no apparent cause other than vascular origin.}

'Financial or Other Competing Interest': None.

Submission 26-11-2017, Peer Review 29-01-2018,

Acceptance 05-02-2018, Published 12-02-2018.

Corresponding Author:

Dr. Amy Susan George,

CKRA 112B, Thyparambil House,

Chakkalamukku, Sreekariyam P. O.,

Trivandrum-695017.

E-mail: amlals1989@gmail.com

DOI: $10.14260 /$ jemds $/ 2018 / 208$

(c) (i) $($ )
The estimated adjusted prevalence rate of stroke range, $84-262 / 100,000$ in rural and $334-424 / 100,000$ in urban areas (2013) in India. The incidence rate is 119 . $145 / 100,000$ based on the recent population-based studies (2013) in India. The risk factors for stroke are modifiable and non-modifiable. The modifiable risk factors are mostly related to the atherosclerotic burden and they include diabetes, hypertension, smoking and hyperlipidaemia. Atherosclerosis is related to various cardiovascular and cerebrovascular events like cerebral infarction. Carotid artery intima media thickness reflects the ongoing process of atherosclerosis in the body. High-resolution B-mode carotid ultrasonography provides a non-invasive method of detecting and measuring arterial wall thickening and the progression of atherosclerosis. There are some studies citing increase in carotid intima media thickness as a prominent risk factor for stroke. The relationship between intima media thickness as a measure of carotid atherosclerosis and the different ischaemic brain infarction subtypes has been the objective of only a few previous studies. Our objective also tries to study their pattern. 


\section{MATERIALS AND METHODS}

The study design was a case control study conducted among patients at General Medicine Department, Medical College, Trivandrum from March 2016. The sample size was calculated based on study by Nikic et al.

A study conducted by Nikic $\mathrm{P}$ et al, "Carotid atherosclerosis, coronary atherosclerosis and carotid intimamedia thickness in patients with ischaemic cerebral disease: Is there any link?" was used to estimate the sample size.

Sample size $N=\frac{\left(Z_{\alpha}+Z_{1-\beta}\right)^{2}\left[\left(\varepsilon_{1}\right)^{2}+\left(\varepsilon_{2}\right)^{2}\right]}{\left(\mu_{1}-\mu_{2}\right)^{2}}=21$

\begin{tabular}{|c|c|c|}
\hline Where $\left(\mathrm{Z}_{\alpha}+\mathrm{Z}_{1-\beta}\right)^{2}$ & $=$ & 10.5 \\
\hline$\alpha$ & $=$ & 0.05 \\
\hline$\beta$ & $=$ & 0.1 \\
\hline$\varepsilon_{1}$ & $=$ & $\begin{array}{l}\text { Standard deviation of carotid intima media thickness } \\
\text { among cases (ischemic stroke) }\end{array}$ \\
\hline$\varepsilon_{2}$ & $=$ & $\begin{array}{l}\text { Standard deviation of carotid intima media thickness } \\
\text { among control. }\end{array}$ \\
\hline$\mu_{1}$ & $=$ & $\begin{array}{l}\text { Mean of carotid intims media thickness among cases } \\
\text { (ischemic stroke). }\end{array}$ \\
\hline$\mu_{2}$ & $=$ & Mean of carotid intima media thickness among control. \\
\hline
\end{tabular}

$2 \mathrm{~N}$ participants were included for meaningful comparison within the groups. Hence, the sample size was taken as 42 cases and 42 controls for the study.

\section{Inclusion and Exclusion Criteria}

Cases were defined as patients admitted with ischaemic strokes diagnosed by NCCT Brain. Controls were defined as patients with negative history for prior or acute onset stroke with normal neurological findings on examination and no previous history of cardiovascular disease. The cases and controls defined as above were included in the study. Patients with acute haemorrhagic stroke, those for whom the quality of ultrasound carotid scanning did not allow for intima media thickness measurement and those who did not cooperate in the ultrasound evaluation of the carotid artery were excluded from the study.

\section{Method of Data Collection}

The data on demographic characteristics, smoking and cardiovascular risk factors were collected as a structured proforma. Carotid intima media thickness of cases and controls were measured using Philips ClearVue 650 4-12 Mhz B Mode Ultrasound Doppler. It was followed by clinical examination, blood parameters, ultrasound measurement of carotid intima media thickness and NCCT Brain. The IMT was measured as the distance between two echoic lines separated by anechoic space. IMT reading was taken avoiding the area of the plaque. The point of measurement was taken $1 \mathrm{~cm}$ proximal to the carotid bulb at the site of maximal thickness. Each carotid was scanned for the presence of plaque and whether any ulcerations are present. In the latest ESH/ESC hypertension guidelines (2013) carotid IMT $>0.9 \mathrm{~mm}$ or presence of plaque had been re-confirmed as a marker of asymptomatic organ damage. As per guidelines, CIMT > $0.9 \mathrm{~mm}$ was considered as risk for ischaemic stroke. Data was analysed using SPSS 16.6 software for windows.
Measurement data was summarised as mean with standard deviation. Chi-square test was used to assess association between CIMT and ischaemic stroke. $\mathrm{P}$ value $<0.05$ was considered significant. Pearson correlation was used to assess correlation between CIMT and size of infarct.

\section{RESULTS}

The mean age among 42 controls were $52.7 \pm 6.9$ and cases $62.7 \pm 8.5$. The mean BMI among cases were $24.8 \pm 2.4$ and among controls were $22.8 \pm 2.8$. Among cases, $50 \%$ had history of diabetes compared to controls where $26.2 \%$ had history of diabetes. History of hypertension was present among $61.9 \%$ of cases and $40.5 \%$ of controls. $35.2 \%$ were current smokers, $21.4 \%$ ex-smokers and $42.9 \%$ non-smokers among cases. Among controls only $16.7 \%$ were current smokers and $7.1 \%$ were ex-smokers, while the majority $76.2 \%$ were non-smokers. Dyslipidaemia was present among $50 \%$ of cases, but only $4 \%$ among controls. History of coronary artery disease was present among $42.9 \%$ of cases, but only among $4 \%$ of controls. History of transient ischaemic attack was present among $7.1 \%$ cases and $4 \%$ of controls. The mean fasting blood sugar among cases were $138.1 \pm 54.9$ and controls were $98.2 \pm 14.1$. The mean total cholesterol was high among cases $198.1 \pm 30.7$ compared to controls $176.3 \pm 25.4$. In the present study, carotid plaques were present in $40.5 \%$ of cases and $14.3 \%$ of controls. The mean carotid intima media thickness in the present study was 0.90 $\pm 0.1 \mathrm{~mm}$ among cases and $0.80 \pm 0.1 \mathrm{~mm}$ among controls, statistically significant at $\mathrm{p}=0.001$. The risk for stroke as in CIMT $>0.9 \mathrm{~mm}$ was present in $57.1 \%$ cases as compared to $23.8 \%$ controls. In our study among infarction subtypes, 52.3\% had Partial Anterior Circulation Infarctions (PACI), 23.8\% had Lacunar Infarctions (LACIs), 16.6\% had Posterior Circulation Infarctions (POCIs) and 7\% had Total Anterior Circulation Infarctions (TACIs). CIMT $>0.9 \mathrm{~mm}$ was found in $68.5 \%$ of PACI, $66.6 \%$ of TACI, $14.2 \%$ of POCI and $60 \%$ of LACI. There was no correlation between carotid intima media thickness and size of infarct in the present study.

\section{Presence of Carotid Plaques among Cases and Controls}

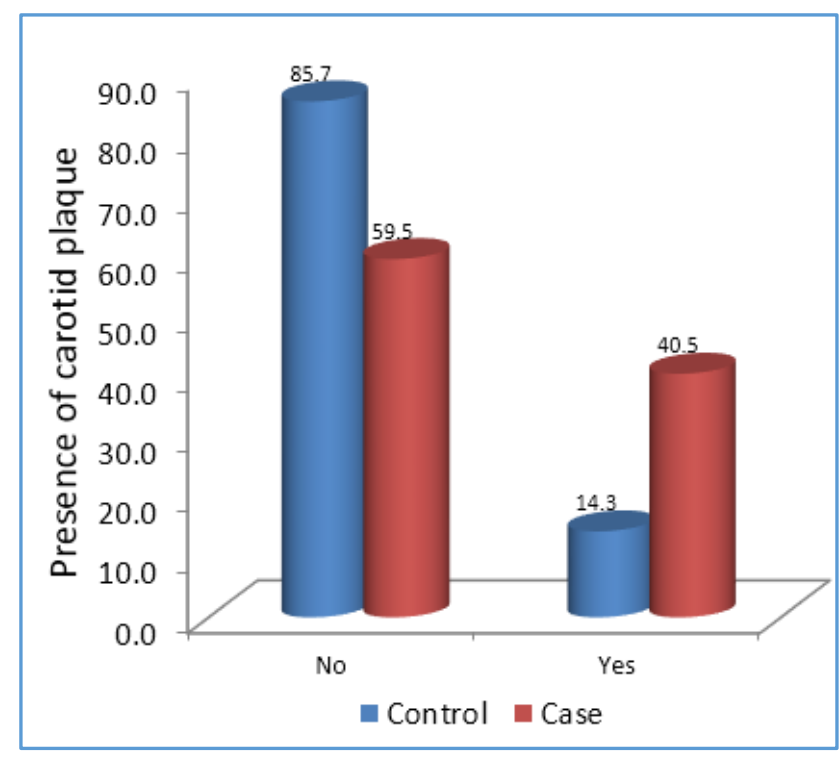

$\mathrm{p}=0.007$ 
Association of Carotid Intima Media Thickness with Ischaemic Stroke

\begin{tabular}{|c|c|c|c|c|c|}
\hline Group & Mean & SD & N & t & p \\
\hline Control & 0.8 & 0.1 & 42 & \multirow{2}{*}{3.32} & \multirow{2}{*}{0.001} \\
\hline Case & 0.9 & 0.1 & 42 & & \\
\hline
\end{tabular}

\section{Risk of Ischaemic Stroke in Cases and Controls}

\begin{tabular}{|c|c|c|c|c|c|}
\hline \multirow{2}{*}{ IMT MEAN } & \multicolumn{2}{|c|}{ Control } & \multicolumn{2}{|c|}{ Case } & Odds \\
\cline { 2 - 5 } & Count & Percent & Count & Percent & (95\% CI) \\
\hline $\begin{array}{c}\text { No risk (CIMT } \leq \\
0.9 \mathrm{~mm})\end{array}$ & 32 & 76.2 & 18 & 42.9 & 1 \\
\hline $\begin{array}{c}\text { Risk (CIMT > } \\
0.9 \mathrm{~mm})\end{array}$ & 10 & 23.8 & 24 & 57.1 & $\begin{array}{c}4.27 \\
(1.67- \\
10.89)\end{array}$ \\
\hline
\end{tabular}

Risk Calculated as Carotid Intima Media Thickness > $0.9 \mathrm{~mm}$ as per ESC Guidelines

\section{Carotid Intima Media Thickness Based on Brain Infarction Subtypes}

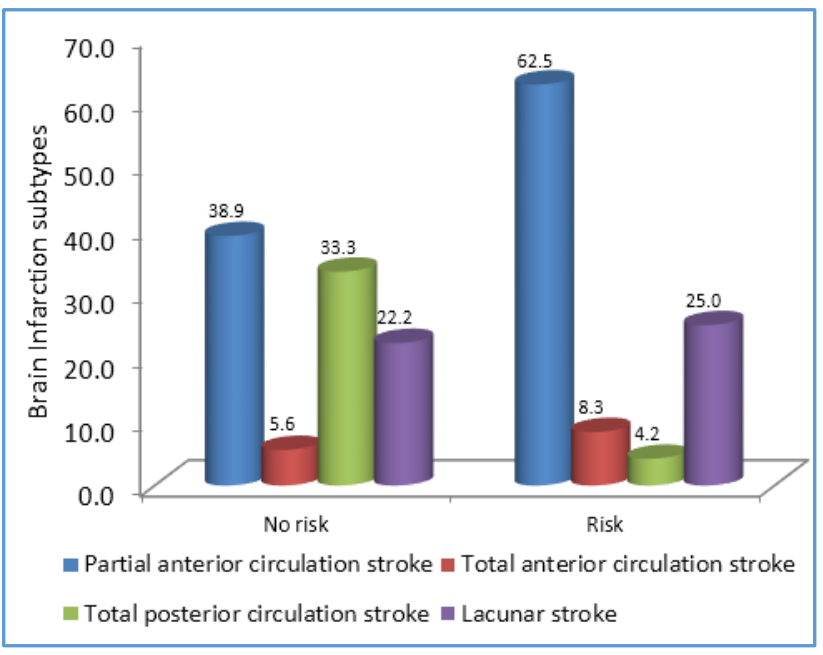

Scatter Diagram for Association of Carotid Intima Media Thickness and Size of Infarct

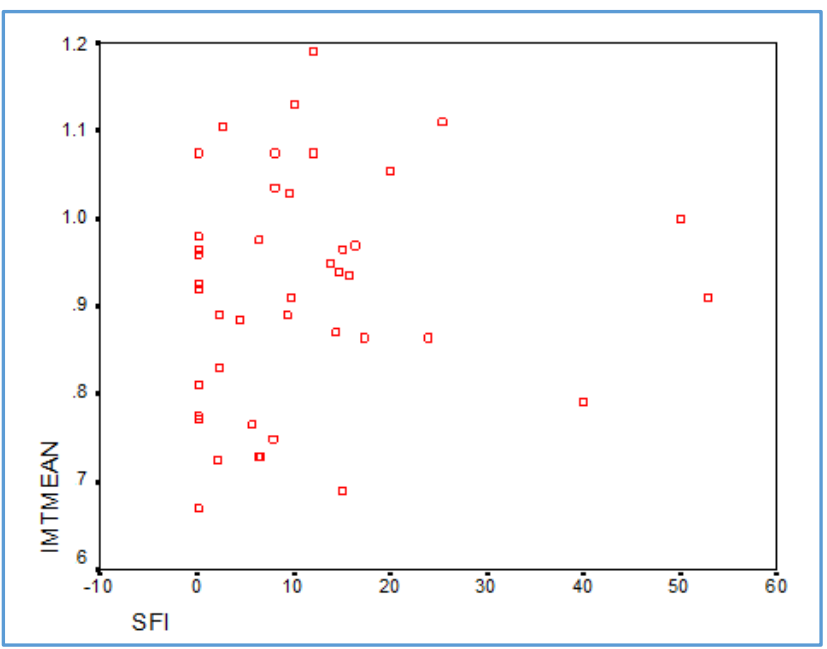

$\mathrm{r}=0.132, \mathrm{p}=0.406$

\section{DISCUSSION}

Stroke is a major disease burden worldwide. Studies have proven that carotid intima media thickness (CIMT) can be used as a marker of atherosclerosis and is a risk factor for cardiovascular diseases. This study tries to find the association between stroke and carotid intima media thickness and also to study the association of CIMT with brain infarction subtypes.

In this study a total of 84 patients, 42 cases and 42 controls were enrolled. Of the total study population, $64.3 \%$ were males and $35.7 \%$ were females. The mean age among cases were $62.7 \pm 8.5$ and controls were $52.7 \pm 6.9$. In a similar study by Sahoo et al,(1) 60 patients and 50 controls were studied. The mean age in the patient group was 62 years and $63.3 \%$ were males. In a study by Harris $S$ et al(2) in Indonesia, a total of 259 patients (78 women and 181 men) were included. The study population was divided into two groups: stroke patients $(n=131)$ and non-stroke patients $(n=$ 128). The total mean age was 56.65 (10.70) years. Mean age was 58.17 (9.88) years old in the stroke group and 55.15 (11.31) years old in the non-stroke group. In comparison to their study, ours showed a higher mean age in patient group. In a study by Nikic $P$ et al,(3) among 75 patients and 21 controls the mean age was $60 \pm 10$ yrs. among study group and $58 \pm 12$ yrs. among controls. The mean age among all subjects were $59 \pm 10$ yrs. as compared to our study where mean age was $57.7 \pm 7.7$ yrs.

In our study, the mean BMI among cases were obtained as $24.8 \pm 2.4$ and among controls were $22.8 \pm 2.8$. Among cases, $50 \%$ had history of diabetes compared to $26.2 \%$ in controls. History of hypertension were present among $61.9 \%$ of cases and $40.5 \%$ of controls. Among cases, $35.2 \%$ were current smokers, $21.4 \%$ were ex-smokers and $42.9 \%$ were nonsmokers. Among controls only $16.7 \%$ were current smokers and $7.1 \%$ were ex-smokers, while the majority $76.2 \%$ were non-smokers. Dyslipidaemia was present among 50\% of cases, but only among $4 \%$ of controls. History of coronary artery disease was present among $42.9 \%$ of cases and among $4 \%$ of controls. History of transient ischaemic attack were present among $7.1 \%$ cases and $4 \%$ of controls. The mean fasting blood sugar among cases were $138.1 \pm 54.9$ and controls were $98.2 \pm 14.1$. The mean total cholesterol was high among cases at $198.1 \pm 30.7$ compared to controls at $176.3 \pm 25.4$. There was significant statistical difference between patient group and control group in terms of above mentioned baseline clinical characteristics. In the GENIC case control study,(4) the baseline clinical characteristics were similar to our study population except for higher age and hypertension. In ARIC prospective study,(5) the relationship between carotid IMT and prevalent cardiovascular disease was studied. The results show increased IMT in participants with prevalent coronary artery disease, cerebrovascular disease and peripheral vascular disease. The carotid IMT in participants with cardiovascular disease was $10 \%$, greater in those with myocardial infarction, $6 \%$ greater in those with angina pectoris, $6 \%$ greater in those with cerebrovascular disease, $19 \%$ greater in those with peripheral vascular disease and $8 \%$ greater in those with any form of cardiovascular disease. In contrast to this the study by Harris $S$ et al,(2) no statistical difference was found among the characteristics of the population across both study groups in 
accordance to hypertension, type 2 diabetes mellitus, dyslipidaemia and smoking history.

In our study, the risk for stroke was $57.1 \%$ among cases as opposed to $23.8 \%$ among controls and was statistically significant. In the study by Nikic $\mathrm{P}$ et al,(3) the prevalence of carotid atherosclerosis in the study group was $80 \%$ versus $28.5 \%$ in the control group. In contrast to our study, their's showed a higher prevalence of carotid atherosclerosis among patient group.

In our study, carotid plaques were present in $40.5 \%$ of cases and $14.3 \%$ of controls. In the study by Sahoo R et al $^{(1)}$ carotid plaques were present in $18(30 \%)$ patients with stroke and 7 (14\%) in controls similar to our study, which showed carotid plaques in 17 cases and 6 controls. In the study by Nikic P et al,(3) there was a significant difference in the distribution of plaque status between cases and controls. Small plaques were detected in $47 \%$ of patients in the study group versus $19 \%$ in the control group similar to our study.

In our study, the mean carotid intima media thickness was found to be $0.90 \pm 0.1 \mathrm{~mm}$ among cases and $0.80 \pm$ $0.1 \mathrm{~mm}$ among controls and was statistically significant at $\mathrm{p}=0.001$. While, in the study by Sahoo $\mathrm{R}$ et al,(1) the mean carotid intima media thickness in the patient group was $0.782 \pm 0.19 \mathrm{~mm}$ and $0.594 \pm 0.98 \mathrm{~mm}$ in control group. Our study showed a higher mean carotid intima media thickness when compared to the above study. There was significant difference between the mean carotid intima media thickness for controls between the two studies with ours showing a higher mean. They used control mean intima media thickness plus two standard deviations as their cut-off. The intima media thickness value thus calculated was $0.79 \mathrm{~mm}$. Of the 60 patients, 29 patients (48\%) had an intima media thickness above $0.79 \mathrm{~mm}$, only 2 subjects (4\%) among the controls had intima media thickness above this cut-off. In our study, cut-off was taken as $0.9 \mathrm{~mm}$ as per ESC guidelines 2013. Out of 42 cases $24(57 \%)$ had intima media thickness above $0.9 \mathrm{~mm}$, while among 42 controls 10 (23.8\%) had intima media thickness above cut-off. In the cross-sectional study by Jain et $\mathrm{al},(6)$ the mean carotid intima media thickness was $0.83 \mathrm{~mm}$ ranging from a minimum of $0.45 \mathrm{~mm}$ to a maximum of 1.096 $\mathrm{mm}$ similar to our study results. The mean intima media thickness in the study by Mukherjee et al(7) from Kolkata was $0.66 \mathrm{~mm}$ in patients with stroke and 0.32 in controls, whereas our study showed a significantly higher mean at $0.9 \mathrm{~mm}$ in cases and $0.8 \mathrm{~mm}$ in controls. In the study by Nikic P et al,(3) the mean carotid intima media thickness was $0.85 \mathrm{~mm}$ in controls versus $1.03 \mathrm{~mm}$ in cases. For all subjects, the mean carotid intima media thickness was $0.99 \mathrm{~mm}$ in their study as opposed to our study at $0.85 \mathrm{~mm}$. As compared to our study, the mean carotid intima media thickness among cases were higher in the study by Nikic P et al.(3) In the GENIC casecontrol study,(4) the mean IMT was $0.797 \mathrm{~mm}$ in stroke patients and $0.735 \mathrm{~mm}$ in controls $(\mathrm{p}=0.0001)$ in comparison with our study which had higher mean intima media thickness among cases and controls.

Our study showed a strong association between carotid intima media thickness and acute ischaemic stroke $(\mathrm{p}=0.002)$. The study by Harris $\mathrm{S}$ et al(2) also showed a significant association between carotid intima media thickness and ischaemic and stroke $(\mathrm{p}=0.008)$ in the Indonesian population. In the Rotterdam Elderly Prospective Study,(8) a case-control study in a subgroup of their population showed an association between common-carotid-artery intima media thickness and the risk of myocardial infarction and stroke in concordance with our study. In a similar study by Jain et al,(6) the most significant finding was that there was a statistically significant increase in the intima media thickness of common carotid artery in patients with CT-proven ischaemic stroke. But their study was cross-sectional and had an inherent selection bias as CIMT was measured only in patients of known ischaemic stroke, while ours was case control study. In the study by Mukherjee et al(7) results indicate that in the common as well as in the internal and external carotid arteries, the intima media thickness is a good predictor of ischaemic stroke similar to our study. But in their study, the sample size was small and the controls were not matched by the risk factors.

In our study, among infarction subtypes $52.3 \%$ had PACIs (Partial anterior circulation infarction), 23.8\% had LACIs (Lacunar infarction), 16.6\% had POCIs (Posterior circulation infarction) and $7 \%$ had TACIs (Total anterior circulation infarction). CIMT $>0.9 \mathrm{~mm}$ was found in $68.5 \%$ of PACI, $66.6 \%$ of TACI, $14.2 \%$ of POCI and $60 \%$ of LACI. There was no correlation between carotid intima media thickness and size of infarct in the present study. According to the study by Nikic $\mathrm{P}$ et al,(3) patients with acute ischaemic brain infarction $(n=75)$ were classified into different subtypes based on the neuroimaging (CT scan) and clinical characteristics (Bamford's criteria). The most frequent subtype was PACI at $45 \%, 25 \%$ had LACIs, $16 \%$ had POCIs and $13 \%$ had TACIs. In our study among infarction subtypes, 52.3\% had PACIs, $23.8 \%$ had LACIs, $16.6 \%$ had POCIs and $7 \%$ had TACIs. In their study $85 \%$ of PACIs had carotid atherosclerosis, while our study showed $68 \%$ which was similar. In the study by Nikic $P$ et al(3) 70\% of TACI had carotid atherosclerosis, while in our study it was $66.6 \%$. Their study showed increased carotid atherosclerosis in $75 \%$ of POCIs, while our study showed only $14.2 \%$. Among LACIs their study showed carotid atherosclerosis among 79\%, while our study showed $60 \%$. Their study showed a significant relationship between intima media thickness and ischaemic brain infarction similar to our study. According to the study by Nikic P et al,(3) there was significant relation between CIMT and ischaemic stroke subtypes, but our study did not find significant relation. In ARIC prospective study,(5) carotid intima media thickness levels were significantly positively associated with risk of each ischaemic stroke subtype: lacunar, non-lacunar and cardioembolic strokes in contrast to our study. In the GENIC case-control study, ${ }^{(4)}$ the difference in carotid intima media thickness between cases and controls were significant in the main subtypes in contrast to our study result. The risk of brain infarction increased continuously with increasing carotid intima media thickness in their study.

\section{CONCLUSION}

Carotid intima media thickness can be used as a tool in assessing risk for ischaemic stroke. In this study, there was significant association between carotid intima media thickness and acute ischaemic stroke. There was no association found between carotid intima media thickness and brain infarction subtypes. There was no association found between carotid intima media thickness and size of infarct among cases. A past history of diabetes, hypertension, 


\section{Jemds.com}

dyslipidaemia, smoking and transient ischaemic attack was found to be a significant risk factor for acute ischaemic stroke. The presence of carotid plaque, high body mass index, high total cholesterol and LDL were found to be significant risk factors for stroke.

\section{REFERENCES}

[1] Sahoo R, Krishna MV, Subrahmaniyan DK, et al. Common carotid intima-media thickness in acute ischemic stroke: a case control study. Neurol India 2009;57(5):627-30.

[2] Harris S. The association of carotid intima-media thickness (cIMT) and stroke: a cross sectional study. Perspect Med 2012;1(1):164-6.

[3] Nikic P, Savic M, Jakovljevic V, et al. Carotid atherosclerosis, coronary atherosclerosis and carotid intima-media thickness in patients with ischemic cerebral disease: is there any link? Exp Clin Cardiol 2006;11(2):102-6.

[4] Touboul PJ, Elbaz A, Koller C, et al. Common carotid artery intima-media thickness and brain infarction: the Étude du Profil Génétique de l'Infarctus Cérébral (GÉNIC) case-control study. The GENIC investigators. Circulation 2000;102(3):313-8.

\section{Original Research Article}

[5] Ohira T, Shahar E, Iso H, et al. Carotid artery wall thickness and risk of stroke subtypes: the atherosclerosis risk in communities study. Stroke 2011;42(2):397-403.

[6] Jain J, Lathia T, Gupta OP, et al. Carotid intima-media thickness and apolipoproteins in patients of ischemic stroke in a rural hospital setting in central India: a cross-sectional study. J Neurosci Rural Pract 2012;3(1):21-7.

[7] Mukherjee SC, Basu AK, Bandyopadhyay R, et al. Correlation of lipid profile and carotid artery plaque as detected by Doppler ultrasound in ischaemic stroke patients--a hospital-based study. J Indian Med Assoc 2006;104(6):325-6, 330.

[8] Bots ML, Hoes AW, Koudstaal PJ, et al. Common carotid intima-media thickness and risk of stroke and myocardial infarction: the Rotterdam study. Circulation 1997;96(5):1432-7. 